\title{
Exploiting natural variation in root system architecture via Genome Wide Association Studies
}

Agnieszka Deja-Muylle ${ }^{1,2}$, Boris Parizot ${ }^{1,2}$, Hans Motte ${ }^{1,2}, T_{0 m}$ Beeckman $^{1,2}$ *

${ }^{1}$ Ghent University, Department of Plant Biotechnology and Bioinformatics, 9052 Ghent, Belgium

2VIB Center for Plant Systems Biology, 9052 Ghent, Belgium

.Corresponding Author: tom.beeckman@psb.vib-ugent.be

Corresponding Author: Tom Beeckman

VIB Center for Plant Systems Biology

Ghent University, Department of Plant Biotechnology

Technologiepark 71

B-9052 Ghent (Belgium)

Tel.: +32 9 3313830; Fax: +3293313809

(C) The Author(s) 2020. Published by Oxford University Press on behalf of the Society for Experimental Biology. All rights reserved. For permissions, please email: journals.permissions@oup.com 


\section{Abstract}

Root growth and development has become an important research topic for breeders and researchers based on a growing need to adapt plants to changing and more demanding environmental conditions worldwide. Over the last few years, Genome Wide Association Studies (GWASs) became an important tool to identify the link between traits in the field and their genetic background. In this review, we give an overview of the current literature concerning GWASs performed on Root System Architecture (RSA) in plants. We summarize which root traits and approaches have been used for GWAS mentioning their respective success rate towards a successful gene discovery. Furthermore, we zoom in on the current technical hurdles in root phenotyping and GWAS and discuss future possibilities in this field of research.

Keywords: Genome Wide Association Studies, Natural variation, Root Development, Root System Architecture, Root Phenotyping, Root Trait, SNP 


\section{Introduction}

Plant roots are of high interest to plant breeders as they are not only responsible for proper anchorage, energy storage and microbial interactions but, most importantly, also for effective water and nutrient acquisition and thus yield efficiency. Root System Architecture (RSA) describes the spatial configuration of a root system in its growing substrate. RSA is determined by the initiation, angularity, and growth of different root subparts (including primary, lateral and adventitious roots), parameters that can vary depending on the genotype (Figure 1) as well as on the environmental conditions. Within the angiosperms, fundamentally different RSAs can be found between mono- and dicotyledonous plants (Bellini et al., 2014). What is, however, common for both groups is that RSA can differ within accessions due to adaptation to environmental conditions (Burridge et al., 2017; Lynch, 2015). In this regard, the study of how certain RSAs can cope with the currently ongoing changing climate is a topic that recently has gained increased scientific attention (Rogers and Benfey, 2015). This renewed interest is further supported by the development of novel phenotyping techniques, facilitating a more in depth characterization of plant's "hidden half" (Atkinson et al., 2019).

Understanding how roots develop in different environments is, however, only part of the whole picture, and increased efforts are made to discover the genes driving RSAs development. This includes Genome Wide Association Studies (GWASs), which basically associate genotype with phenotype information in a large population that is characterized by a natural variability of traits or a panel of cultivar species. Recently, GWAS became applicable to an increasing number of plant species due to the advent of Next Generation Sequencing. GWAS uses a set of accessions belonging to a species under study and searches for statistic association between a given trait and Single Nucleotide Polymorphisms (SNPs). Essentially, it tests whether two groups, having each a specific genetic polymorphism, are phenotypically different, and as such tests the association between a trait and a locus (Liu and Yan, 2019; Ogura and Busch, 2015). As neighboring regions of associated loci can be inherited together, i.e. in Linkage Disequilibrium (LD), more loci than only the associated SNP can be associated to the phenotype. In comparison to QTL mapping, GWAS has a much wider range of variability as it investigates not only two parental lineages, but makes use of different ecotypes that have evolved over time and adapted to different environments (Korte and Farlow, 2013). As such, 
a GWAS may be used instead of fine-mapping to identify genes underlying QTLs (Motte et al., 2014; Sterken et al., 2012).

In this review, we will first discuss different phenotyping strategies used for RSA analysis followed by an overview of the progress being made in our understanding of RSA by applying GWAS to the model species Arabidopsis as well as multiple crop species. An overview of the GWAS experiments on RSA can be found in Figure 2.

\section{Phenotyping: a prerequisite for GWAS}

An important step for GWAS is the proper collection of phenotypic data. The analysis of RSA traits has evolved significantly slower than other traits, due to the limited accessibility inherent to the, in general, below-ground growth of roots. During the last few years, improvements of techniques to image RSA in soil and/or on growing media combined with more efficient image analysis tools to generate quantifiable traits, gave rise to an increased interest in RSA studies (Atkinson et al., 2019). Depending on the phenotyping technique, different parameters of RSA can be assessed and therefore included in GWAS. The broad range of phenotyping approaches described in literature (Kuijken et al., 2015) are prompted by the choice of plant species, the traits of interest and the specific environmental conditions or developmental phases under study. Depending on the species and the complexity of the RSA, dynamic parameters can also be visualized and measured with the help of time-lapse imaging and automatic root detection algorithms (York and Lobet, 2017). An overview of root traits submitted to GWAS is provided in Figure 2.

Arabidopsis is a model plant very often used for GWAS, due to the availability of a huge set of sequenced accessions, which are easy to grow and to propagate. To assess the RSA of Arabidopsis, in vitro conditions are often used, such as growing seedlings on plates with gelfilled media. This allows a relatively easy imaging and analysis of the RSA thanks to the growth of the roots on the transparent medium. Arabidopsis can also be grown using hydroponics and aeroponics but these techniques alter some traits such as angularity or overall shape (Kuijken et al., 2015). A more natural condition can be achieved by the use of soil-filled rhizotrons, consisting of specific boxes with transparent front panels, filled with appropriate growth substrate, that facilitate observation of root growth over time. For Arabidopsis, improvements were made by transforming accessions with a luminescence expressing construct, facilitating 
imaging and analysis of the otherwise difficult to detect thin roots in a GLO-Root system (Rellán-Álvarez et al., 2015). Overall, there are some limits to the characterization of plant RSA using Arabidopsis. Due to its very thin roots and increased root tangling at older stages of plant development, studies in pots in greenhouse conditions are still hampered, and precise root tracing can mostly only be performed in two dimension and by hand. This limits the analysis to a small number of samples and/or to very young and untangled root systems. Additionally, translation and validation of discoveries made in model species such as Arabidopsis to crops of agricultural value is not straightforward. Crops on the other hand have much bigger roots allowing easier extraction from the growing substrate and more convenient scoring of root traits. For many years, shovelomics, a method to extract and visually score root traits of mature crown root systems, has been used in breeding programs. Above mentioned rhizotrons are also increasingly implemented for growing crop species such as maize or rice. Even if the read-out remains limited to only two dimensions, which differs from the more complex 3D environment of the soil, this system is the closest to mimicking real life conditions. Crop root systems can also be phenotyped in pots with the help of computed tomography (CT) scanning, in transparent tubes, or using paper rolls. The bigger size and complexity of their genome, additional to the moderate availability of genetic tools for gene manipulation, have delayed the use of GWAS in crop species. However, with the progress in sequencing technologies and improvement in genome annotation, GWAS on crops becomes more accessible.

\section{GWAS as a tool to find fundamental RSA genetic pathways}

GWAS can be used to pinpoint any type of gene, either genes involved in developmental pathways or genes only activated in certain growth conditions. Figure 3 provides an overview of different conditions that have been addressed in GWASs to search for genetic loci involved in the conditional modulation of root growth and RSA.

One of the first attempts to get insight into RSA genetics via GWAS was performed in Arabidopsis and applied an allometric method for quantification (Rosas et al., 2013). More specifically, a dataset of variability in size and shape was generated by mapping a set of developmental mark-points (e.g. primary root tip, first lateral root position) on each root image. By doing so, landmark-based morphological root models were created, giving 
information on how lateral roots are distributed along the primary axis, allowing to extract information further used for Principal Component Analysis. Two Principal Components (PCS) explaining most of the variability in root size and allometry were used for GWAS and two genes were detected: Phosphate 1 (PHO1) and Root System Architecture 1 (RSA1). Interestingly, subjecting mutants of these genes to different treatments showed an interaction between genotype and environment. This suggests that the allelic variance in these genes not only underlines natural variation in RSA, but also contributes to the plastic responses of the root. The same study compared natural variation and plastic responses in a set of 69 Arabidopsis accessions, and observed that the variation within individual accessions is similar to the range of variation observed in an accession under different treatments. This further corroborates the existence of genes controlling both phenotypic plasticity and natural variation, and implies a role of root plasticity in adaptive change.

Also specific developmental pathways in root development can be subjected to GWAS. A study of Meijón et al. (2013) analyzed root traits at the cellular level such as root cell length and size of the root apical meristem in 96 Arabidopsis accessions. This resulted in the identification of KURZ UND KLEIN 1 (KUK1), an F-box gene regulating meristem and cell length in roots, thus having influence on primary root (PR) length and root system depth, a desired trait for water uptake efficiency in plant breeding. Slovak et al. (2019) performed a GWAS on root growth rate and 15 other root traits on a panel of 252 natural accessions of Arabidopsis and found mutiple distinct associations. Authors focused on root growth rate and on two of the top associations, and using a T-DNA line candidate approach identified and characterized one gene, Arabidopsis Adenylate Kinase 6 (AAK6) which natural allelic variation is involved in root growth rate determination

Rice has been one of the first crops studied via means of association mapping (Clark, 2010). One of the recent rice studies was performed on 18 root traits from a panel of indica and japonica accessions, using a soil column system (Phung et al., 2016). The strongest associations were found for root depth, thickness, and number of crown roots. Next to a vast number of new candidate genes, some of the genes identified in this study are known root developmental genes. As such, the auxin signaling gene OsIAA4 or the cell cycle gene $C y c D 6 ; 1$ important for root meristem formation, were identified together with 22 other candidate genes that need further validation. 
An interesting trait investigated in a rice GWAS, is the "root cone angle" which is a proxy for the volume of soil that can be explored by the root system. More specifically, this trait was analyzed in "Rhizoscopes", specifically designed rhizoboxes containing glass beads, a hydroponic solution and a grid of nails to hold the root system in place (Bettembourg et al., 2017). In this study, both an indica and japonica panel were used and yielded in total 65 associations. Based on a meta-analysis, 17 interesting genes were selected, including F-box proteins, receptor kinases and auxin responsive genes. In another study, eight root traits, also including the angle of the roots in different zones, were characterized on 93 rice accessions grown in aerobic systems consisting of plastic mesh baskets (Biscarini et al., 2016). Among associated SNPs, four have been in LD with known loci involved in root morphology, for example a QTL controlling parameters such as root thickness and number of tillers in drought stress. This illustrated that sampling a relative small number of accessions can still lead to relevant associations. In another study, an extended set of 795 rice accessions was scored for only two traits, root length and thickness (Zhao et al., 2018). Transcriptomic analysis between few accessions with most robust-root (long root) and the ones with non-robust-root (short root), allowed to narrow down the list of candidate genes. Known genes were identified such was WOX11, indole-3-acetic acid amino synthetase OsGH3.1, RICE SALT SENSITIVE3 (RSS3), showing that a vast dataset with fewer traits scored can also be efficient in gene detection. Wheat has only recently been used in association studies, previously hindered by the lack of a proper reference genome and its tetraploid or hexaploid nature. A panel of durum wheat including recombinant inbred lines and 183 cultivars grown on vertical black screening plates filled with filter paper have been investigated (Maccaferri et al., 2016). Different RSA traits were measured together with seminal root traits, shoot length and plant dry weight. Moreover, this study combined the GWAS with a linkage mapping in two populations to prioritize some regions of special interest with regard to RSA, that can be used as potential candidates for marker assisted selection. Since limited overlap in results was found between the two techniques, especially for the root growth angle, the necessity of using both to achieve a more in-depth candidate gene discovery is emerging. Interestingly, some root phenotypes of contrasting accessions grown for longer periods in field studies could be repeated, indicating that phenotyping at younger stages can be a legitimate proxy for later stages traits. 
Recently, root traits of a population of 201 hexaploid wheat seedlings grown in a paper-roll supported hydroponic system were also subjected to GWAS (Beyer et al., 2019). Multiple candidate genes, selected based on proximity to the SNP hit and supported by literature data, have been described. Subsequently, the effect of allelic variation in known Reduced Height (Rht) genes and in the 1R/1B translocation event on the root phenotype was investigated via means of genotyping. Presence of one of the alleles, Rht-B1b, in the 201 accessions, appeared to decrease root dry matter, while presence of Rht-D1b allele increased seminal root length and root diameter. Maize has also been studied via means of GWAS. One study investigated 20 root traits but also shoot parameters on a panel of 384 maize lines grown in paper-roll system (Pace et al., 2015). The study reports 263 possibly interesting polymorphisms, 17 of them associating with multiple traits. One gene, predicted to code a putative protein 1-phospatidylinositol-4-phosphate 5-kinase, was linked to root diameter and root surface area and showed a high expression in both shoots and roots, making it an interesting target. Another study investigated RSA traits in 300 double haploid maize lines, derived from 66 landraces (Sanchez et al., 2018). Seven SNPs were associated with both root and shoot seedling traits. Multiple SNPs were in LD with previously reported QTLs for RSA parameters. All results await further validation. For rapeseed, association mapping panel has been scored for seven root traits in greenhouse conditions (He et al., 2019). Out of 295 candidate genes, eight genes were found to have Arabidopsis homologues with a described function in root development. Furthermore, based on available genetic and protein interaction data from model plants, protein network interaction dataset for all genes of interest was constructed. 113 genes were linked in protein-protein interaction networks; seven genes were located in key positions of the same large cluster, indicating their possible important role as interactors of other associated genes. A role in root hair formation was previously showed for the Arabidopsis homologue of one of these genes, PFT1.

In soybean landraces, four loci have been associated to lateral root number and root thickness distribution (Prince et al., 2019). The most significant loci pointed at an unknown gene on chromosome 16, associated with lateral root number. Further allele mining revealed two polymorphic sites, pinpointing at protein sequence changes, that still need further validation. 
GWAS can also be used to detect common genetic pathways controlling RSA between species (Zheng et al., 2019). Maize and sorghum diversity panels were phenotyped with a specifically developed phenotyping platform allowing for manual excavation and compressedair-based root cleaning combined with a semi-automated pipeline for the extraction of RSA traits from images. For maize, 107 significant SNPs were associated with six RSA traits, representing 77 genes. 62 additional RSA-associated maize genes were identified via eRDGWAS (expression read depth genome-wide association study) that makes use of gene expression levels (RNA seq data from root tips of germinating seedlings) to link them to the phenotypic traits. Interestingly, $16 \%$ of the identified RSA-associated maize genes were earlier reported to affect RSA in other species which is a high confirmation rates and encourages to further explore the RSA-associated genes in this study for potential use in crop improvement. Furthermore, authors have shown a substantial overlap among the RSA-associated genes detected in maize and sorghum and seven pairs of syntenic maize and sorghum genes could be identified to be associated with RSA traits, which is significantly more overlap than would be expected by chance. Moreover, GWAS in maize resulted in a higher resolution (up to single gene or near single gene) as compared to Sorghum arguing for strategies to use GWAS in one species in order to identify candidate genes within large chromosomal windows in other crops.

Despite difficulties of GWAS in crop species, the approach has been successful in finding associations in many species as well as in Arabidopsis. Genes of versatile nature have been linked to fundamental role in RSA development. Their further biological validation can pave the way to more efficient breeding programs in crop improvements.

\section{GWAS on RSA upon environmental stresses}

Drought is a major threat for crops and becomes even more pronounced with the climate change. Some plants/accessions have however evolved different mechanisms to cope with water limitation, which makes them a material of choice to perform GWAS and understand better resistance mechanisms. Two GWASs have been performed on rice, which is a species with high water requirements. In one study, 529 rice accessions have been phenotyped for 21 RSA traits related to root length/deeper rooting, root volume and root weight, in drought stress and normal conditions (Li et al., 2017). More than $85 \%$ of the associated loci identified by GWAS were aligned with previously reported QTL regions 
responsible for root morphology. Moreover, GWAS identified 11 root-related genes, including DEEPER ROOTING 1 (DRO1), the WUSCHEL-related homeobox WOX11 and the rice ortholog of PINOID (OsPID). Two candidate genes underwent further functional analysis. NARROW LEAF1 (NAL1) was associated to the dry weight of the deep roots in drought conditions. Genetic complementation, using both the NAL1 promotor and the coding sequence of an accession with higher deep root weight, into a weakly performing background, resulted in a greater root volume, confirming NAL1 as the causal gene. Next to the phenotype, also a difference in expression level between the two lines was complemented. Likewise, overexpression lines were also characterized by a larger RSA. Another gene that underwent further investigation is JASMONATE ZIM-domain 1 (OsJAZ1). OsJAZ1 was associated with the dry weight of the shallow roots in normal conditions. Here, a local association analysis revealed only one highly associated SNP, which was located in the promoter region of the gene. Overexpression of the gene led to an increased number of crown roots and higher root elongation rate and confirmed the link between the expression of OsJAZ1 and the root trait. The second study focused on drought conditions and used an exhaustive list of 35 traits including root morphology and anatomy near the root-shoot junction, but also shoot morphology, biomass values and plant physiological traits (Kadam et al., 2017). All traits have been evaluated under normal and drought conditions, and additionally, the phenotypic plasticity was defined as the relative change between the two conditions. Among the candidate genes, many had been reported earlier to play a role in root development. For instance SCARECROW (key transcription factor in the root meristem, involved in, amongst others, radial root growth), associated to root length with a certain diameter size in control conditions, and to the late metaxylem diameter, in drought conditions; SMALL AUXIN UPREGULATED RNA 3 (OSSAUR3, known to regulate root elongation through auxin metabolism or possibly by stimulating PM H+-ATPase activity (Spartz et al., 2014)) associated to root volume in control conditions; LONESOME HIGHWAY (LHW, controls the stele and xylem development) associated to late metaxylem number in control conditions; and Gibberellic Acid-Stimulated 10 (GASA10, known to be involved in phytohormone crosstalk and to regulate root growth), associated to root volume and few other traits in drought conditions. Also associations related to the plasticity of root traits towards drought stress were detected and identified 41 candidate genes, with CLAVATA1, a receptor kinase involved in root stem-ness (Stahl et al., 2013); and two PIN genes, involved in auxin transport. 
Other species have also been evaluated upon drought or osmotic stress. Wheat has been studied for root depth aspects only, whereas maize and barley were also evaluated on shoot parameters. In maize, GWAS investigated 396 maize lines grown in soil-filled minirhizotrons with water deficit (Zaidi et al., 2016). Both structural root traits and agronomic traits were phenotyped, which was possible due to growth up to reproductive stage. Many associations have been detected for the root traits, including three that associated to root volume and dry weight, water use and transpiration efficiency. For wheat, a GWAS was performed on root length of accessions grown in a hydroponic solution with or without PEG to induce osmotic stress (Ayalew et al., 2018). In barley, accessions have been phenotyped in pot systems with specific irrigation under well-watered and drought stress conditions (Reinert et al., 2016). Few associations have been found in both experiments but further validations still need to be achieved.

Salinity stress influence on RSA was studied via means of GWAS for Arabidopsis and few crops. Plant root growth is very susceptible to salinity stress upon which it reacts by redistributing biomass between the primary, the lateral roots and the shoot (Negrão et al., 2016). Julkowska et al (2017) have studied 347 Arabidopsis accessions under two different $\mathrm{NaCl}$ concentrations. Among the 100 loci associated to Salt-induced changes in RSA, two candidate genes were further validated: CYTOCHROME P45O FAMILY 79 SUBFAMILY B2 (CYP79B2), and HIGH AFFINITY $K^{+}$TRANSPORTER 1 (HKT1). CYP79B2 was associated with average LR length and $H K T 1$ with the ratio average LR length/main root length. Validation of the loss of function mutant line indicated that CYP79B2 is necessary to maintain the lateral root growth under salinity conditions, but is not essential under control conditions. In contrast, over-expression lines of HKT1 reduced LR formation in stress conditions. Additionally, authors showed that HKT1 presents a high allelic variation in its promoter region possibly pointing at evolutionary mechanisms towards plant tolerance to salinity. Another salinity study has been performed in Arabidopsis using hydroponics system. 200 accessions, grown in both low and high salt stress conditions, were scored for primary root length and average relative root length (Kobayashi et al., 2016). Multiple genes involved in known related pathways showed associations. However, only few genes were represented in both conditions, possibly indicating that response to low and high stress might be governed by distinct pathways. 
Interaction of plants with pathogens can also contribute to modification of root architecture. In cowpea, associations were found for overall RSA ideotypes, for higher seed yield and pod number and Striga tolerance (Burridge et al., 2017). This was performed using a diversity panel of 189 lines phenotyped via shovelomics. A GWAS performed on a total of 19 RSA and shoot parameters resulted in a list of 32 associated loci, several of them co-localizing with previously identified QTL regions linked to developmental features such as seed weight, seed number, pod number and Striga tolerance. The link between pathogen resistance and RSA was also investigated on pea (Desgroux et al., 2018). In this instance, 10 RSA traits have been phenotyped on 266 pea lines grown in a greenhouse. Additionally, three resistance related traits were phenotyped in a controlled chamber. One loci, most probably pointing at a MAP-kinase, has been detected to associate with both the root projected area and pathogen resistance. Alleles responsible for higher projected area could as such potentially lead to lower disease score.

\section{GWAS on RSA traits scored in micro- and macronutrient deficiencies}

Micro and macronutrient-uptake by roots is essential for plant development. A high plasticity in RSA is observed in response to nutrients availability, to optimize the acquisition of these resources that are often heterogeneously distributed in the soil (Jin et al., 2017; Motte et al., 2019). Ideotypes of RSA structure have been proposed that optimize the most efficient nutrient acquisition (White et al., 2013). Several GWASs have been successfully performed to unravel mechanisms of RSA adaptation to nutrients. Bouain et al. (2018) investigated the response to low or high concentration of zinc on 231 accessions grown on supplemented medium. PR length was measured and two SNPs were detected for -Zn condition but none for $+Z n$. One of the associated genes of interest, AZELAIC ACID INDUCED (AZI1), was further shown to be differentially expressed between stress conditions. Regulatory regions of the $A Z I 1$ gene presented a high level of polymorphism between contrasting accessions showing long or short $P R$ in -Zn environments. Loss of function mutants shown a significantly shorter PR than the wild-type Col-0 phenotype in -Zn conditions while overexpression lines had longer PR. Using a similar set-up but for iron (Fe) deficiency, the impact of its availability on Arabidopsis root growth in 134 accessions has been investigated (Satbhai et al., 2015). Multiple associations were found for total root length and linked to shorter roots in -Fe conditions. Based on differential expression levels between conditions of candidate genes, in the DNA region 
around the significant SNP, FERRIC REDUCTION OXIDASE 2 (FRO2) was further investigated. A complementation analysis of fro 2 mutant in Col-0 background with a specific haplotype from a contrasting accession, showed that the allelic variation in a non-coding region of FRO2 is causal for the significantly shorter roots in -Fe conditions. In another study, dedicated this time to iron stress, Li et al. (2019) have analyzed primary root length at high Fe and Fe tolerance (root length in high Fe relative to the control on normal medium) among 319 natural accessions. GWAS yielded only one significant peak that exceeded the Bonferroni-corrected significance threshold of $5 \%$, common for both the absolute root length or relative root length at high Fe phenotypes. In silico gene expression analysis for 10 genes surrounding the lead SNP pointed at S-nitrosoglutathione reductase (GSNOR), and authors showed its requirement for high Fe tolerance using knockout lines and allelic complementation. In a study performed by Lee et al (2017), GWAS was used to study rice tolerance towards zinc deficiency. Total root number, whole plant and grain biomass were estimated. 43 of the resulting associated genes have been selected based on their differential expression in $\mathrm{Zn}$ treatments. One gene, Os06g44220, associated to all traits and was strongly upregulated upon stress conditions in both root and shoot showing that GWAS has the power to detect genes involved both in above and underground plant development pathways.

Two main macronutrients treatments, nitrogen and phosphate, have also been included in associations studies. For nitrogen, 96 accessions of Arabidopsis have been screened on two different concentrations (Gifford et al., 2013). GWAS on seven root traits, revealed that only $1 / 3$ of the associating genes were commonly detected for both nitrogen treatments and were associated to the same trait (average lateral root length). Based on expression patterns of the candidate genes, in seven accessions representing the most contrasting root architectures between different conditions, the list of candidates was narrowed to 13 genes. Mutants of one of these genes, JASMONATE RESPONSIVE 1 (JR1), that associated to the lateral root difference in low and high nitrogen, and to lateral root length in low nitrogen, displayed shorter LRs average lengths in low nitrogen treatments. A second gene, associating with shorter LRs in low, but not in high concentrations of nitrogen, was $P h z C$, a gene responsible for Phenazine protein biosynthesis. Those two genes impact the phenotype in specific environments only, showing the power of GWAS to detect genes involved in condition specific pathways. 
Since phosphate is an immobilized nutrient in the top soil, the adaptation of RSA plays an important role in capturing it. Different phosphate regimes were used independently to study 19 root traits in sorghum, in sand-filled rhizotrons (Parra-Londono et al., 2018). GWAS detected seven associations in high $\mathrm{P}$, and one in low $\mathrm{P}$ conditions. The distribution of root hairs, an interesting root trait but strictly speaking not an element of RSA, was also studied using phosphate treatments (Stetter et al., 2015). Based on gene ontologies, two genes had a possible relation to root development. Root hairs play a pivotal role in $\mathrm{P}$ uptake process as they increase the root uptake surface. The GWAS analysis included 166 accessions grown on split growth medium plates, with $\mathrm{P}$ available only in the top part. Increased root hair density (in low phosphorus conditions) was observed mostly for genotypes with shorter and more scarce root hairs in normal conditions. The GWAS identified the RECEPTOR LIKE PROTEIN 48 (RLP48) associating with root hair density and CYTOKININ RESISTANT 1/ ETHYLENE INSENSITIVE 2 (CKR1/EIN2) and ALTERNATIVE OXYDASE 1D (AOX1D) associating with root hair surface. Loss-of-function mutants showed increased root hair densities corroborating the role of these genes in the process. WRKY 6 was also detected as a candidate gene and local finemapping in a more limited number of accessions led to identification of multiple significant SNPS in the coding region of WRKY6. The loss-of-function mutants not only showed an increase in root hair density but also root hair length.

Roots growing in soil are never subjected to only one type of stress. Kawa et al. (2016) analyzed root growth in phosphate deprived conditions combined with additional salt stress. The authors made use of the observation that the inhibiting effects of mild salinity stress on root traits can, partially, be overcome by the introduction of Pi starvation. 17 RSA traits were scored upon salt stress, low $P$, or a combination of both for 330 accessions. Most of the GWAS associations were detected for the double stress experiments and correlated to the lateral root length trait. Few accessions deviated from the patterns of RSA traits responses to stress. These accessions are interesting case studies to address the genetic variability in response to stress conditions. Another example of GWAS detecting genes involved in root growth under multi-nutrient deprivation has recently been performed by Bouain et al (2019). Apart from phosphate, zinc and iron deficiency, -Pi/-Fe and -Pi/-Zn combinations were analyzed. 277 accessions have been grown in-vitro. Normalized root growth rate (RGR) values (ratio of RGR for each condition divided by RGR in control) were used to perform GWAS. 145 genes were 
detected and only two overlapping regions were identified among different treatments, more precisely between -P and -Zn, comprising in total 10 genes. Most significant SNPs were located in the coding region of a member of the ABI5-binding proteins, described previously to be responsible for stress response in germinating seeds through ABA signaling. Using a systems approach that combines the GWAS with network-based candidate identification and reverse genetics, 3 genes were validated to be involved in responses to combined stress: VARIANT IN METHYLATION 1 (VIM1), FORMIN-LIKE-PROTEIN-6 (FH6) and VOLTAGE-DEPENDENT ANIONSELECTIVE CHANNEL PROTEIN 3 (VDAC3). Expression levels of those genes in WT and 8 accessions with contrasting RGR, were measured to confirm their role in RGR alteration, making it the first GWAS to identify genes involved in multi-stress root responses. Finally, an informative pattern of RGR emerged from this study: deficiencies in respectively Fe or Zn cause reductions in RGR that could be minimalized by introducing the second stressor $-P$ deficiency. Interestingly, the widely used Col-0 accession was classified as not representative for RGR responses to phosphate and zinc conditions, similar to the findings of its response to hormonal treatments (see further, Ristova et al, 2018).

\section{Phytohormonal treatment}

The direct influence of phytohormones on RSA has been investigated in a GWAS on Arabidopsis, where ten RSA traits were investigated upon abscisic acid (ABA), cytokinins (CK), and auxin (IAA) treatment (Ristova et al., 2018). ABA had a strong negative influence on the traits related to LRs (such as density, number or average length), while IAA modulated those traits positively. On the other hand, IAA had a negative influence on the PR growth rate. CK had a milder negative effect on overall root growth such as: PR growth rate, lateral root number and length of lateral roots. Those responses could, however, differ amongst accessions. Interestingly Col-0 was one of the few accessions deviating the most from the overall trend of above mentioned responses. This observation suggests that Col-0, though commonly used as a reference accession, it is not an ideal representative to study root plasticity under phytohormonal treatments. Amongst 114 associations detected for the three independent treatments, one was shared among them all. Overall, the large part of associated loci directed to new, undescribed genes. A more recent GWAS study was able to link cytokinin with the distribution of the root system (Waidmann et al., 2019). For 210 Arabidopsis accessions, the Gravitropic Set-point Angle (GSA), the angle with respect to the gravity vector 
at which an organ is maintained as a consequence of gravitropism, was determined for individual LRs and proved to vary among accessions by even $40^{\circ}$. Data on GSA of LRs was used to perform GWAS and one significant SNP, located in the CYTOKININ OXIDASE 2 (CKX2) gene, was further investigated. By means of CKX2 loss-of-function and overexpression lines in Col-0 background, a more horizontal growth and increased bending were observed, respectively, indicative for a role in GSA. The authors also could demonstrate that the identified SNP affects the activity of the CKX2 enzyme due to altered signal peptide processing in different accessions and concluded that variation in signal peptide processing of CKX2 contributes to the natural variation of CK-dependent angular LR growth in Arabidopsis. Another recent study has shed light onto how auxin is regulating root gravitropic responses and RSA depth (Ogura et al., 2019). 215 accessions were tested for eight RSA traits in presence of the auxin transport inhibitor NPA (1-N-Naphthylphthalamic acid). A strong significant association for the root angle trait resulted from the GWAS. The tag SNP was located in the EXOCYST7OA3 gene, and subsequent haplotype analysis, transcription expression profiling and validation using mutant and overexpression lines, proved that this gene is involved in root responses to gravity stimulus. It influences PIN4 localization and consequently auxin levels in the columella cells. Furthermore, analysis of accessions with contrasting haplotypes, climate parameters in the sites of origin, RSA in soil conditions, and fitness under water limiting conditions, indicated that the identified gene may belong to a locus responsible for the development of shallower root systems, providing possible adaptation and increased fitness to environments where temporary water deficit occurs. The examples here discussed, show that GWAS can be applied to find genes responsible for different RSA traits. Depending on the parameters and environment of the experiment it can not only look at the adaptive differences accumulated within strain of one species over time, but also it can point to genes activated in plasticity responses to various conditional environmental stresses that differ among accessions.

\section{Conclusions and perspectives}

The success of a GWAS depends on the design of the experiments and mostly three aspects: (i) the correct investigation of the research question, which in this case is linked to precise and in-depth phenotyping (including the conditions used, the phenotyping techniques and quantification of the traits etc.), (ii) a sufficient sample size and structure, and finally, (iii) the biological validation (Ogura and Busch, 2015). In case of RSA architecture, advances in 
phenotyping techniques as well as dedicated image analysis algorithms have expanded over time the range of root traits that can be measured. The first phenotyping experiments were mostly limited to traits such as PR length, number of lateral roots and biomass. In following studies, authors have already been able to tackle more elaborated traits related to the distribution of the root system, such as shape, root system angularity, bushiness, root distribution in different zones, diameters of different root parts, convex hull or root volume. The progresses made in extracting those more complicated traits also allow to score elaborated RSA traits at later stages of plant development. Additionally, the cultivation methods used for phenotyping are influencing the type of traits that will be extracted. For example, in this review, GWAS on RSA upon salinity stress in Arabidopsis has been undergone using two different growing techniques (plates and hydroponics), which allow the collection of different traits but might explain why different significant associations were detected (Figure 2). It is also important to investigate as many RSA traits as a phenotyping technique allows. Different root traits have different phenotypic plasticity span, so some traits will present a higher variability than others upon changed environments. Thus, investigation of some traits might not lead to significant associations because of a lack of variability in the population.

A second crucial choice in GWAS set-up is the sample size. The population size determines the power of GWAS via phenotypic and genotypic variability. The current review describes populations sizes ranging from 69 accessions in the case of a study in Arabidopsis to 795 for another study in rice (Figure 2). There is no universal recipe on how many samples should be taken into account while performing GWAS. This mostly depends on the species population structure, the type of traits investigated, the traits variability span in the given species etc. It is, however, commonly believed that increasing the number of accessions grants more chances of saturation of the dataset with the trait of interest (Ogura and Busch, 2015). This is because most traits are governed by a large number of loci with small or moderate effect. That is a crucial part of nature of GWAS as it struggles to detect alleles with low frequencies or epistasis effect in the population and so increasing the sample size increases the power of detection (Liu and Yan, 2019). In the above-mentioned examples, authors have been successful in finding genes of interest even in small populations, indicating that probably the corresponding traits are governed by a small number of loci with higher effect. Some of 
the presented studies, despite investigating the same traits, failed to find the same associations (e.g. phosphate limitation in Arabidopsis or drought stress in rice) which can be explained by differences in the population panels, that create a versatile genetic background where some loci can be more prone to be detected than others (Figure 2). Structure of the accessions panel can also have influence on the resulting gene detection. Sampling of accessions worldwide, from diverse environments is very common, as it allows to increase genetic and phenotypic variance. However, at the same time population structure issues in such panels can become a greater problem in confounding the analysis. Another approach has been to collect samples locally, for example accessions from one country, allowing to lower the heterogeneity. On the other hand, alleles that are relevant for shaping phenotypic diversity at a global scale might not occur at a high enough frequency to be picked up by GWAS (Korte and Farlow, 2013).

Thirdly, the selection and validation of candidate genes within the LD window of the significant associations is an important final step to complete the GWAS. In case of Arabidopsis studies, many genes detected have been validated. KUK1, EXOCYST7OA3 and AZI1 have a direct or indirect influence on root depth and thus drought resistance. Some have been validated and linked to lateral root traits such as $J R 1$ and $P h z C$ in nitrogen stress and CYP79B2 and CYP79B3 in saline conditions. FRO2 in iron deficiency have been associated with the total root length. In some cases, genes already characterized for non-root related functions have been associated, validated and linked to RSA traits. For instance, WRKY6 transcription factor is known to be interacting with PHO1, determining phosphate nutrition. It was identified in GWAS and loss of function mutants showed defects in root hair densities in low P. Also HKT1 was known to have a role in plant development upon salinity stress but after GWAS it was showed to directly reduce the lateral root formation in salt stress conditions (Figure 2). The possibility to find and validate several genes for RSA is made easier in Arabidopsis as it is characterized by a small, sequenced genome with freely accessible information, high SNP coverage and multiple genetic tools available for validation (Korte and Farlow, 2013). The validation is more complex when it comes to crops, and many of the above described GWAS yielded multiple loci that have not been fully validated yet. There are however indications that these genes are involved in root development processes based on transcription levels in the root, on overlap with previously detected genes or QTLs, on gene ontologies or on the 
existence of gene orthologues with related functions in other species. The lack of molecular tools and ease of experimentation, however, hampers the validation of genes detected in crops. Cross-population validation is nowadays introduced to verify candidate genes in a different population but it is time consuming. Also Crisper-Cas is a promising tool that might help validation (Liu and Yan, 2019). Post-GWAS analyses in crop species are now focusing on overlapping multiple loci detected in independent studies thereby making use of regulatory and interaction networks and implementing functional characterization (Gupta et al., 2019). In this review, only two studies, performed in rice, led to the validation of candidate hits (Figure 2); NAL1 gene was associated with deep roots dry weight upon drought; and OsJAZ1 for root dry weight in normal conditions and four novel genes related to root and shoot lengths in hydroponics (Li et al., 2017; Zhao et al., 2018). Few known genes were detected in crops, such as SCARECROW or WOX11, but still lack validation in respect to their direct implication with the studied trait (Figure 2). On the other hand, these experiments have also produced multiple sets of marker trait associations, giving rise to huge datasets of QTLs which can be already used for breeding strategies, such as marker assisted selection (Gupta et al., 2019).

The power of GWAS lies in that it can detect mutations causative for a deregulation in gene expression but also can give significant associations in regions responsible for example for protein structure and that can modulate substrate or protein interaction affinity, also post transcriptional modifications, etc. This expands the possibilities of gene-trait association but makes it also more difficult to validate the results. Among genes detected in the mentioned studies are for instance: transcription factors, auxin related kinases, polar auxin transporters, binding proteins and various transporters. These genes belong to multiple cellular and biological processes, indicating that RSA development is a complicated process, involving many different and intertwining pathways, that are sometimes hard to be dissected and attributed to single traits. In comparison with other techniques such as mutagenesis, GWAS seems then a perfect tool to face this complexity and discover new genes (Liu and Yan, 2019). It's also important to mention that genes detected in GWASs described in this review are very often not the most prominent parts of known root development pathways. Those genes have been previously discovered, usually via traditional forward genetics, such as mutant screens. One main reason being that mutagenesis experiments are prone to detect genes with the 
biggest effects, while GWAS is population sensitive and the variability in the genetic background of a whole panel can give more significance to other genes involved in the pathway (Ogura and Busch, 2015).

In conclusion, this review summarizes recent GWASs that have been performed on RSA, and summarized in Figure 2. This type of analysis can be used to investigate RSA genetic determinants of traits that evolved through generations. Different approaches were successful in detecting genes of interest, affecting RSA either in normal conditions, but also upon treatments mimicking environmental changes. Future efforts in this field will focus on; further improvements of underground root phenotyping and visualization; improving how GWAS can overcome its elemental drawbacks such as confounding effects of pleiotropy, epistasis or synthetic associations; and finally developments in the molecular and bioinformatics toolboxes necessary for the validation of the detected genes (Liu and Yan, 2019, Gupta et al., 2019). 


\section{Glossary}

GWAS - Genome-wide association study, a study of a genome-wide set of genetic variants in different individuals to see if any variant is associated with a trait.

Ideotype - the idealized appearance of a plant variety or organ. In the present it used for roots.

LR - lateral root, a root that develops on the primary root as a branching root.

Linkage Disequilibrium (LD) - measures the degree to which two loci are associated. In a given population, two loci are in LD when their presence is non-random, in contrast to the occurrence that is expected from if they were independent.

PR - primary root, the first root that originates during embryogenesis and develops from the radicle of the germination embryo.

RSA - the spatial configuration of a root system in the soil, used to describe the shape and structure of root systems.

SNP - single-nucleotide polymorphism, a substitution of a single nucleotide that occurs at a specific position in the genome.

Split growth medium plates - Split agar plates contain 2 different growth media and are made to reveal local effects of e.g. nutrient or osmotic conditions on root growth.

tag SNP - SNP that is representative for a wider genomic region (in LD with that region). It marks a particular haplotype in that region and abolish the need of genotyping all SNPs in that region. In practice, GWAS is initially only testing tag SNPs.

LD window - region around tag SNP that has been flagged in GWAS as a significant one. Genes located in that region are considered for further analysis as candidate genes.

Principal Component Analysis - statistical procedure allowing to reduce dimensions by reducing a large set of variables to a small set that still contains most of the information and can be associated to a certain portion of variation explained. 


\section{Figure Legends}

Fig. 1: Examples of different RSAs in three accessions of Arabidopsis thaliana, in soil-filled rhizotrons, illustrating the prominent genetic differences in RSA. Plants are 24 DAG. A - Col-0, B - Wc-1, C-Had$1 b$

Fig. 2: Overview of publications cited in this review as well as associated root traits phenotyped, main experiment characteristics, and main findings. Illustration of root traits: A- Total Root Length, BPrimary Root Length, C-Lateral Root Traits, D-Total Number of Roots, E-Root Diameter Traits, F-Seminal and/or Crown Root Traits, G-Width and/or Depth of the RSA, H-RSA Bushiness (Maxiumum/Median number of roots), I-Root Length Distribution, J-Root Surface Traits, K-Root Volume, L-RSA Allometry, M-Root Angles, N-Root Growth Rates, O-Root microscopical Traits and Root Hair Traits, P-Root and/or Shoot Biomass, Q-Shoot and/or Grain Traits.

Fig. 3: Examples of factors and/or growth conditions modulating root development and RSA. External (environmental) and endogenous (phytohormones) cues discussed in this review are represented.

\section{Acknowledgements}

We thank both reviewers for their constructive comments on the manuscript and Maria Fransiska Njo for help with artwork. Work in the Beeckman lab was supported by grants G002817N [Bilateral Research Cooperation with the Chinese Ministry of Science and Technology (MOST) 2016YFE0109900], G022516N, G020918N, and G024118N from the Research Foundation-Flanders (FWO) and a BOF-ZAP grant from the Special Research Fund of the Ghent University. 


\section{REFERENCES}

Atkinson JA, Pound MP, Bennett MJ, Wells DM. 2019. Uncovering the hidden half of plants using new advances in root phenotyping. Current Opinion in Biotechnology 55, 1-8.

Ayalew H, Liu H, Börner A, Kobiljski B, Liu C, Yan G. 2018. Genome-Wide Association Mapping of Major Root Length QTLs Under PEG Induced Water Stress in Wheat. Frontiers in plant science $\mathbf{9}$, 1759-1759.

Bellini C, Pacurar DI, Perrone I. 2014. Adventitious Roots and Lateral Roots: Similarities and Differences. Annual Review of Plant Biology 65, 639-666.

Bettembourg M, Dardou A, Audebert A, Thomas E, Frouin J, Guiderdoni E, Ahmadi N, Perin C, Dievart A, Courtois B. 2017. Genome-wide association mapping for root cone angle in rice. Rice (New York, N.Y.) 10, 45-45.

Beyer S, Daba S, Tyagi P, Bockelman H, Brown-Guedira G, Mohammadi M, IWGSC. 2019. Loci and candidate genes controlling root traits in wheat seedlings-a wheat root GWAS. Functional \& Integrative Genomics 19, 91-107.

Biscarini F, Cozzi P, Casella L, Riccardi P, Vattari A, Orasen G, Perrini R, Tacconi G, Tondelli A, Biselli C, Cattivelli L, Spindel J, McCouch S, Abbruscato P, Valé G, Piffanelli P, Greco R. 2016. GenomeWide Association Study for Traits Related to Plant and Grain Morphology, and Root Architecture in Temperate Rice Accessions. PLOS ONE 11, e0155425.

Bouain N, Korte A, Satbhai SB, Nam HI, Rhee SY, Busch W, Rouached H. 2019. Systems genomics approaches provide new insights into Arabidopsis thaliana root growth regulation under combinatorial mineral nutrient limitation. PLoS Genet. 15, e1008392.

Bouain N, Satbhai SB, Korte A, Saenchai C, Desbrosses G, Berthomieu P, Busch W, Rouached H. 2018. Natural allelic variation of the AZI1 gene controls root growth under zinc-limiting condition. PLoS Genet. 14, e1007304.

Burridge JD, Schneider HM, Huynh B-L, Roberts PA, Bucksch A, Lynch JP. 2017. Genome-wide association mapping and agronomic impact of cowpea root architecture. Theoretical and Applied Genetics 130, 419-431.

Clark RM. 2010. Genome-wide association studies coming of age in rice. Nature Genetics 42, 926.

Desgroux A, Baudais VN, Aubert V, Le Roy G, de Larambergue H, Miteul H, Aubert G, Boutet G, Duc G, Baranger A, Burstin J, Manzanares-Dauleux M, Pilet-Nayel M-L, Bourion V. 2018. Comparative Genome-Wide-Association Mapping Identifies Common Loci Controlling Root System Architecture and Resistance to Aphanomyces euteiches in Pea. Frontiers in plant science 8, 2195-2195.

Gifford ML, Banta JA, Katari MS, Hulsmans J, Chen L, Ristova D, Tranchina D, Purugganan MD, Coruzzi GM, Birnbaum KD. 2013. Plasticity Regulators Modulate Specific Root Traits in Discrete Nitrogen Environments. PLOS Genetics 9, e1003760.

Gupta PK, Kulwal PL, Jaiswal V. 2019. Chapter Two - Association mapping in plants in the post-GWAS genomics era. In: Kumar D, ed. Advances in Genetics, Vol. 104: Academic Press, 75-154. 
He Y, Hu D, You J, Wu D, Cui Y, Dong H, Li J, Qian W. 2019. Genome-wide association study and protein network analysis for understanding candidate genes involved in root development at the rapeseed seedling stage. Plant Physiology and Biochemistry 137, 42-52.

Jin K, White PJ, Whalley WR, Shen J, Shi L. 2017. Shaping an Optimal Soil by Root\&\#x2013;Soil Interaction. Trends in Plant Science 22, 823-829.

Julkowska MM, Koevoets IT, Mol S, Hoefsloot H, Feron R, Tester MA, Keurentjes JJB, Korte A, Haring MA, de Boer G-J, Testerink C. 2017. Genetic Components of Root Architecture Remodeling in Response to Salt Stress. The Plant Cell 29, 3198-3213.

Kadam NN, Tamilselvan A, Lawas LMF, Quinones C, Bahuguna RN, Thomson MJ, Dingkuhn M, Muthurajan R, Struik PC, Yin X, Jagadish SVK. 2017. Genetic Control of Plasticity in Root Morphology and Anatomy of Rice in Response to Water Deficit. Plant Physiology 174, 2302-2315.

Kawa D, Julkowska MM, Sommerfeld HM, ter Horst A, Haring MA, Testerink C. 2016. PhosphateDependent Root System Architecture Responses to Salt Stress. Plant Physiology 172, 690-706.

Kobayashi Y, Sadhukhan A, Tazib T, Nakano Y, Kusunoki K, Kamara M, Chaffai R, luchi S, Sahoo L, Kobayashi M, Hoekenga OA, Koyama H. 2016. Joint genetic and network analyses identify loci associated with root growth under $\mathrm{NaCl}$ stress in Arabidopsis thaliana. Plant, Cell \& Environment 39, 918-934.

Korte A, Farlow A. 2013. The advantages and limitations of trait analysis with GWAS: a review. Plant methods 9, 29-29.

Kuijken RCP, van Eeuwijk FA, Marcelis LFM, Bouwmeester HJ. 2015. Root phenotyping: from component trait in the lab to breeding. Journal of experimental botany $66,5389-5401$.

Lee J-S, Sajise A, Gregorio G, Kretzschmar T, Ismail A, Wissuwa M. 2017. Genetic dissection for zinc deficiency tolerance in rice using bi-parental mapping and association analysis. TAG. Theoretical and applied genetics. Theoretische und angewandte Genetik 130.

Li, B., Sun, L., Huang, J., Göschl, C., Shi, W., Chory, J., \& Busch, W. 2019. GSNOR provides plant tolerance to iron toxicity via preventing iron-dependent nitrosative and oxidative cytotoxicity. Nature communications, 10(1), 1-13.

Li X, Guo Z, Lv Y, Cen X, Ding X, Wu H, Li X, Huang J, Xiong L. 2017. Genetic control of the root system in rice under normal and drought stress conditions by genome-wide association study. PLOS Genetics 13, e1006889.

Liu H-J, Yan J. 2019. Crop genome-wide association study: a harvest of biological relevance. The Plant Journal 97, 8-18.

Lynch JP. 2015. Root phenes that reduce the metabolic costs of soil exploration: opportunities for 21st century agriculture. Plant, Cell \& Environment 38, 1775-1784.

Maccaferri M, El-Feki W, Nazemi G, Salvi S, Canè MA, Colalongo MC, Stefanelli S, Tuberosa R. 2016. Prioritizing quantitative trait loci for root system architecture in tetraploid wheat. Journal of experimental botany $67,1161-1178$.

Meijón M, Satbhai SB, Tsuchimatsu T, Busch W. 2013. Genome-wide association study using cellular traits identifies a new regulator of root development in Arabidopsis. Nature Genetics 46, 77. 
Motte H, Vanneste S, Beeckman T. 2019. Molecular and Environmental Regulation of Root Development. Annual Review of Plant Biology 70, 465-488.

Motte H, Vercauteren A, Depuydt S, Landschoot S, Geelen D, Werbrouck S, Goormachtig S, Vuylsteke M, Vereecke D. 2014. Combining linkage and association mapping identifies RECEPTOR-LIKE PROTEIN KINASE1 as an essential Arabidopsis shoot regeneration gene. Proceedings of the National Academy of Sciences 111, 8305-8310.

Negrão S, Schmöckel SM, Tester M. 2016. Evaluating physiological responses of plants to salinity stress. Annals of Botany 119, 1-11.

Ogura T, Busch W. 2015. From phenotypes to causal sequences: using genome wide association studies to dissect the sequence basis for variation of plant development. Current Opinion in Plant Biology 23, 98-108.

Ogura T, Goeschl C, Filiault D, Mirea M, Slovak R, Wolhrab B, Satbhai SB, Busch W. 2019. Root System Depth in Arabidopsis Is Shaped by EXOCYST70A3 via the Dynamic Modulation of Auxin Transport. Cell 178, 400-412.e416.

Pace J, Gardner C, Romay C, Ganapathysubramanian B, Lübberstedt T. 2015. Genome-wide association analysis of seedling root development in maize (Zea mays L.). BMC Genomics 16, 47.

Parra-Londono S, Kavka M, Samans B, Snowdon R, Wieckhorst S, Uptmoor R. 2018. Sorghum rootsystem classification in contrasting $P$ environments reveals three main rooting types and rootarchitecture-related marker-trait associations. Annals of botany 121, 267-280.

Phung NTP, Mai CD, Hoang GT, Truong HTM, Lavarenne J, Gonin M, Nguyen KL, Ha TT, Do VN, Gantet P, Courtois B. 2016. Genome-wide association mapping for root traits in a panel of rice accessions from Vietnam. BMC Plant Biology 16, 64.

Prince SJ, Valliyodan B, Ye H, Yang M, Tai S, Hu W, Murphy M, Durnell LA, Song L, Joshi T, Liu Y, Van de Velde J, Vandepoele K, Grover Shannon J, Nguyen HT. 2019. Understanding genetic control of root system architecture in soybean: Insights into the genetic basis of lateral root number. Plant, Cell \& Environment 42, 212-229.

Reinert S, Kortz A, Léon J, Naz AA. 2016. Genome-Wide Association Mapping in the Global Diversity Set Reveals New QTL Controlling Root System and Related Shoot Variation in Barley. Frontiers in plant science 7, 1061-1061.

Rellán-Álvarez R, Lobet G, Lindner H, Pradier P-L, Sebastian J, Yee M-C, Geng Y, Trontin C, LaRue T, Schrager-Lavelle A, Haney CH, Nieu R, Maloof J, Vogel JP, Dinneny JR. 2015. GLO-Roots: an imaging platform enabling multidimensional characterization of soil-grown root systems. eLife 4, e07597.

Ristova D, Giovannetti M, Metesch K, Busch W. 2018. Natural genetic variation shapes root system responses to phytohormones in Arabidopsis. The Plant journal : for cell and molecular biology 96, 468-481.

Rogers ED, Benfey PN. 2015. Regulation of plant root system architecture: implications for crop advancement. Current Opinion in Biotechnology 32, 93-98.

Rosas U, Cibrian-Jaramillo A, Ristova D, Banta JA, Gifford ML, Fan AH, Zhou RW, Kim GJ, Krouk G, Birnbaum KD, Purugganan MD, Coruzzi GM. 2013. Integration of responses within and across 
Arabidopsis natural accessions uncovers loci controlling root systems architecture. Proceedings of the National Academy of Sciences 110, 15133-15138.

Sanchez DL, Liu S, Ibrahim R, Blanco M, Lübberstedt T. 2018. Genome-wide association studies of doubled haploid exotic introgression lines for root system architecture traits in maize (Zea mays L.). Plant Science 268, 30-38.

Satbhai SB, Ristova D, Busch W. 2015. Underground tuning: quantitative regulation of root growth. Journal of experimental botany 66, 1099-1112.

Slovak R, Setzer C, Roiuk M, Bertels J, Göschl C, Jandrasits K, Beemster G.T. and Busch W, 2019. Ribosome assembly factor Adenylate Kinase 6 maintains cell proliferation and cell size homeostasis during root growth. New Phytologist. doi.org/10.1111/nph.16291.

Spartz AK, Ren H, Park MY, Grandt KN, Lee SH, Murphy AS, Sussman MR, Overvoorde PJ, Graya WM. 2014. SAUR Inhibition of PP2C-D phosphatases activates plasma membrane H+-ATPases to promote cell expansion in Arabidopsis. Plant Cell 26, : 2129-2142.

Stahl Y, Grabowski S, Bleckmann A, Kühnemuth R, Weidtkamp-Peters S, Pinto Karine G, Kirschner Gwendolyn K, Schmid Julia B, Wink René H, Hülsewede A, Felekyan S, Seidel Claus AM, Simon R. 2013. Moderation of Arabidopsis Root Stemness by CLAVATA1 and ARABIDOPSIS CRINKLY4 Receptor Kinase Complexes. Current Biology 23, 362-371.

Sterken R, Kiekens R, Boruc J, Zhang F, Vercauteren A, Vercauteren I, De Smet L, Dhondt S, Inzé D, De Veylder L, Russinova E, Vuylsteke M. 2012. Combined linkage and association mapping reveals CYCD5; 1 as a quantitative trait gene for endoreduplication in Arabidopsis. Proceedings of the National Academy of Sciences of the United States of America 109, 4678-4683.

Stetter MG, Schmid K, Ludewig U. 2015. Uncovering Genes and Ploidy Involved in the High Diversity in Root Hair Density, Length and Response to Local Scarce Phosphate in Arabidopsis thaliana. PLOS ONE 10, e0120604.

Waidmann S, Ruiz Rosquete M, Schöller M, Sarkel E, Lindner H, LaRue T, Petřík I, Dünser K, Martopawiro S, Sasidharan R, Novak O, Wabnik K, Dinneny JR, Kleine-Vehn J. 2019. Cytokinin functions as an asymmetric and anti-gravitropic signal in lateral roots. Nat Commun., 10: 3540. doi: 10.1038/s41467-019-11483-4.

White PJ, George TS, Gregory PJ, Bengough AG, Hallett PD, McKenzie BM. 2013. Matching roots to their environment. Annals of botany 112, 207-222.

York L, Lobet G. 2017. Phenomics of root system architecture: Measuring and analyzing root phenes. The Plant Cell 29, tpc.117.tt0917.

Zaidi PH, Seetharam K, Krishna G, Krishnamurthy L, Gajanan S, Babu R, Zerka M, Vinayan MT, Vivek BS. 2016. Genomic Regions Associated with Root Traits under Drought Stress in Tropical Maize (Zea mays L.). PLOS ONE 11, e0164340.

Zhao Y, Zhang H, Xu J, Jiang C, Yin Z, Xiong H, Xie J, Wang X, Zhu X, Li Y, Zhao W, Rashid MAR, Li J, Wang W, Fu B, Ye G, Guo Y, Hu Z, Li Z, Li Z. 2018. Loci and natural alleles underlying robust roots and adaptive domestication of upland ecotype rice in aerobic conditions. PLOS Genetics 14, e1007521. 


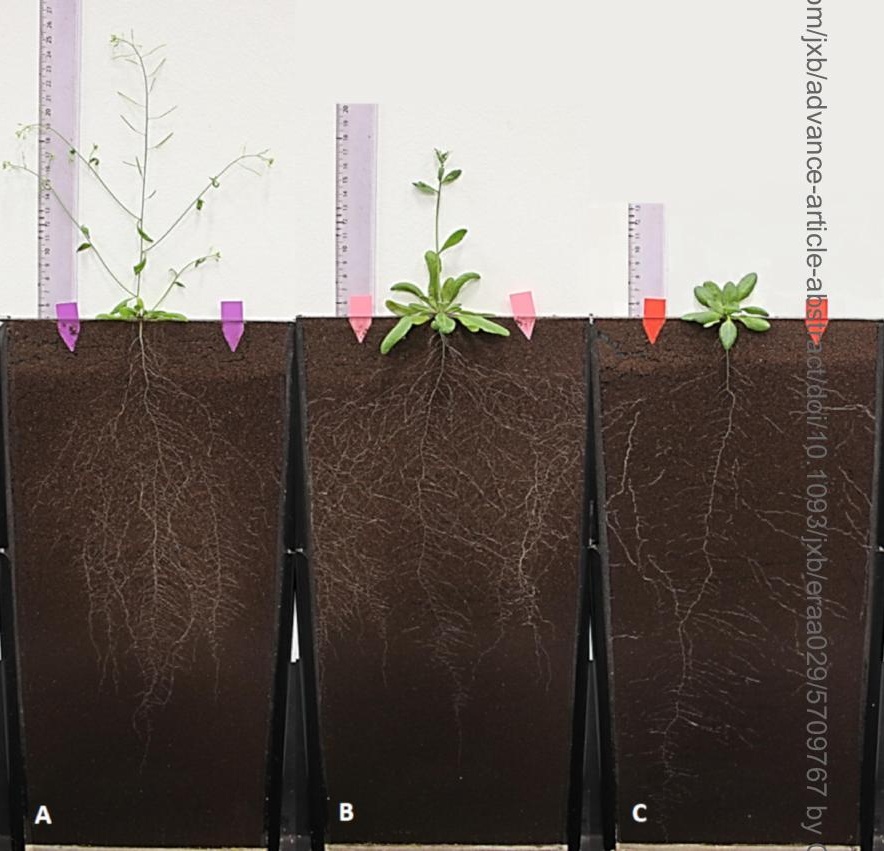




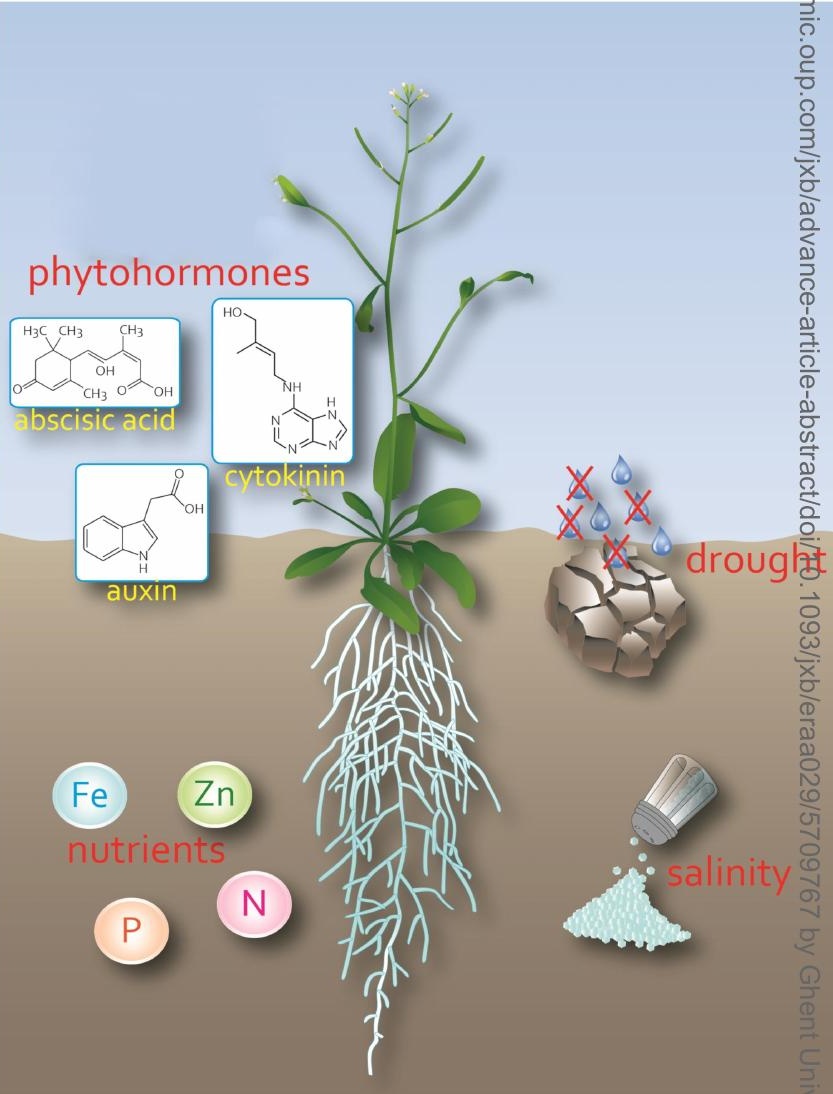




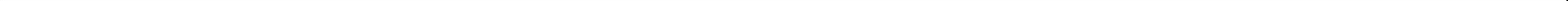

